\title{
Management of patients suspected of having dengue fever: Association of Illness severity with Clinical parameters: A single center experience from Southern Sri Lanka
}

\author{
Karunatilake $\mathrm{H}^{1}$, Wijesinghe $\mathrm{CJ}^{2}$, Wijenayake $\mathrm{HGK}^{3}$, \\ Wettasinghe SS ${ }^{4}$, Mendis MTK ${ }^{5}$, Niroshan WGT ${ }^{6}$
}

\begin{abstract}
Introduction

Dengue has been associated with significant mortality and its management is governed mainly by clinical experience and consensus of experts than evidence. This study was carried out to assess the association of disease severity and clinical parameters of patients suspected of having dengue fever.
\end{abstract}

\section{Method}

A retrospective descriptive study was conducted in District General Hospital (DGH), Matara using hospital data available in patients' records. A total of 1005 adult patients with fever and low platelets admitted during the months of May, June July and August 2017 were studied. Trends in white cell count (WCC), Platelets counts, haematocrit (PCV) and Platelet distribution width (PDW) were recorded. Comparisons were made between the days of the illness, drop in platelet count, rise in the PCV\&WCC and change in PDW, among Dengue fever (DF) and Dengue hemorrhagic fever (DHF) patients. Correlation with disease severity with the presence of comorbidities and NS1 antigen positivity was also assessed.

\section{Results}

No correlation was found with disease severity and presence of comorbidities or NS1 antigen positivity. Rise in PCV \& WCC and drop in platelet counts were noted with the onset of leaking phase of DHF.

\section{Conclusions}

Rapid drop in platelet counts appears to be the most reliable parameters to indicate the onset of leaking phase. Previously described $20 \%$ rise of PCV is not a reliable indicator of the onset of leaking in patients hospitalized in the early phase of the illness.

Keywords: Dengue fever, Dengue Shock Syndrome, Plasma leakage, Epidemics, Sri Lanka

\section{Introduction}

Dengue is a Flavivirus causing a spectrum of illness that range from fever like disease, dengue fever (DF) to dengue hemorrhagic fever (DHF) which can progress to dengue shock syndrome (DSS) and death. ${ }^{1}$ Infection

1. Consultant Physician, General Medical Unit, District General Hospital, Matara

2. Senior Lecturer, Department of Community of Medicine, Faculty of Medicine, University of Ruhuna

3,4,5,6 Medical Officer, General Medical Unit, District General Hospital, Matara

Corresponding Author: Harindra Karunatilake, email: sunrat21@gmail.com

https://orcid.org/0000-0002-8306-2594 with dengue virus imperils about 20 million people every year in tropical and subtropical countries. ${ }^{1}$ The mortality rate is around $1-2 \%$. The case fatality of severe dengue in Asian countries is around 0.5-3.5\%. ${ }^{1}$

In recent times dengue has caused major epidemics in Sri Lanka, 2017 recording the highest magnitude with significant morbidity and mortality. ${ }^{2}$ In the absence of a specific anti-viral therapy, the clinical treatment of dengue fever largely relies on prompt and judicious fluid management and frequent monitoring. Many consensus guidelines stress on the importance of fluid resuscitation particularly during the critical phase where too much and too little both can do equally harm. ${ }^{3}$

Plasma leakage heralds the onset of critical phase in DHF and rarely progress to DSS. Plasma leakage occurs due to increased capillary permeability, which is tran- 
sient and lasts around 24-48 hours. 4 Once the plasma leakage is considerable, it can be detected by presence of free fluid on ultra sound examination of the abdomen. Guidelines stress the importance of identifying the onset of the critical phase when plasma leakage ensues. This is when appropriate fluid therapy and frequent monitoring should be initiated. It is paramount that all available clinical and hematological parameters and their trends are utilized to identify the onset of the critical phase of the illness.

For this study, we assessed the clinical parameters and their trends recorded during management of patients admitted with suspected dengue fever to District General Hospital, Matara during the 2017 epidemic. The aim of the study was to correlate the onset of critical phase with the duration of the illness, drop in platelet count, rise in the haematocrit/packed cell volume (PCV) and the white cell count (WCC), and compare DF and DHF patients based on distribution of these parameters. We also looked at the platelet distribution width (PDW) in both groups, to see any correlation with the onset of critical phase and the change in PDW. Further, we assessed whether antigen positivity and presence of comorbidities has any relationship with the occurrence of DHF.

\section{Materials and methods}

\section{Study design \& setting:}

This was a retrospective descriptive study conducted using hospital data available in patients' records. The study was conducted in District General Hospital (DGH), Matara-major referral hospital in Matara district, in the Southern Province of Sri Lanka.

\section{Participants:}

The study included all patients admitted to general medical units of the District General Hospital (DGH), Matara with fever and low platelets during the months of May, June July and August 2017. A total of 1005adult patients with suspected dengue fever (patients with fever and low platelet count who underwent ultrasound scanning to confirm/exclude leaking phase of the illness) were selected for the study. Patients who were transferred to other institutions after admission, patients who were admitted directly to the intensive care units and paediatric patients(age less than 16 years) with suspected dengue fever were excluded from the study.

Patients present with fever and reduction in platelet counts (<150 x 103 per $\mathrm{ml}$ ) were considered as suspected dengue fever (DF) and the presence of fluid in the abdominal cavity on ultrasound examination categorized patient as a case of dengue hemorrhagic fever (DHF).
Data collection:

Records of all eligible patients available at DGH Matara were retrieved from the hospital record room. Serial clinical parameters were extracted and recorded in a pre-tested data extraction form designed for this purpose. Data were extracted and recorded by the investigators and/or trained medical officers. Approval for data collection was obtained from the Director, DGH Matara.

Statistical analysis:

All data were coded and entered into a database created using IBM SPSS statistical software for analysis. Comparisons were made between the days of the illness, drop in platelet count, rise in the haematocrit (PCV) \& the white cell count and change in PDW, among DF and DHF patients

The platelet counts just before and after the onset of leaking were used to calculate the percentage drop in platelet counts. In the same way, rise in Packed Cell Volume (PCV) was calculated using respective PCV values before and after the onset of critical phase. In non DHF patients, who did not have ultrasound evidence of fluid leaking, the highest magnitude drop in platelet counts and the highest rise in PCV in serial full blood counts were considered for comparison of percentage drop in platelet count and percentage rise in PCV/WCC. In addition, the trend in WCC and PDW were also compared in both groups. Unusual values suspected to be due to laboratory errors were excluded from the analysis.

Mann-Whitney $U$ test was used for comparison of haematological parameters, since the variable showed a skewed distribution. Antigen positivity, when available and presence of co-morbidities was also compared between the two groups using Chi-square test and Fisher's exact test to examine association with the fluid leakage.

The ethical clearance for the study was obtained from the Ethical Review Committee, Faculty of Medicine, Galle.

\section{Results}

$56 \%$ of the 1005 sample was male. The study sample was a relatively young with a mean age of 34 years (Mean \pm SD $=36.9 \pm 14.3$ years). Most patients were admitted after four days of fever (Mean $\pm S D=4.02 \pm 1.4$ days; median $=4$ days). Serological tests were not done in most patients as it was not available in the hospital. Less than $15 \%$ of patients (143) had the Dengue NS1 antigen test done, which was positive in $13.5 \%$ (136)patients. Presence of co-morbidities was seen in a minority (10.0\%) (Table 1$)$. We did not assess the antibody status of the patients. 
Table 1. Disease related characteristics of the sample $(n=1005)$

\begin{tabular}{|c|r|r|}
\hline Characteristics & Number & Percentage \\
\hline Category of disease & & \\
\hline Dengue fever (DF) & 586 & 58.3 \\
\hline Dengue haemorrhagic fever (DHF) & 419 & 41.7 \\
\hline On admission & 131 & 13.0 \\
\hline In ward & 288 & 28.7 \\
\hline & & \\
\hline Presence of co-morbidities & & \\
\hline No reported co-morbidities & 905 & 90.0 \\
\hline Co-morbidities present & 100 & 10.0 \\
\hline Diabetes mellitus & 64 & 6.4 \\
\hline Hypertension & 71 & 7.1 \\
\hline Ischemic heart disease & 8 & 0.8 \\
\hline Bronchial asthma & 55 & 5.5 \\
\hline
\end{tabular}

Table 2. Distribution of NS1 antigen status and co-morbidities in DF and DHF patients

\begin{tabular}{|c|c|c|c|c|c|}
\hline \multirow{2}{*}{\multicolumn{2}{|c|}{ Characteristics }} & \multicolumn{2}{|c|}{ Category of disease } & \multirow{2}{*}{$\begin{array}{c}\text { Total } \\
\text { No (\%) }\end{array}$} & \multirow{2}{*}{$\begin{array}{c}P \\
\text { value }\end{array}$} \\
\hline & & $\begin{array}{c}\text { DF } \\
\text { No (\%) }\end{array}$ & $\begin{array}{c}\text { DHF } \\
\text { No (\%) }\end{array}$ & & \\
\hline \multicolumn{6}{|l|}{ NS1 antigen status ( $n=143$ ) } \\
\hline Positive & & $73(53.7 \%)$ & $63(46.3 \%)$ & $136(100.0 \%)$ & \multirow{2}{*}{1.000} \\
\hline Negative & & $4(57.1 \%)$ & $3(42.9 \%)$ & $7(100.0 \%)$ & \\
\hline \multicolumn{6}{|c|}{ Presence of co-morbidities ( $n=181$ ) } \\
\hline \multirow{2}{*}{ Diabetes mellitus } & Present & $37(57.8 \%)$ & $27(42.2 \%)$ & $64(100.0 \%)$ & \multirow{2}{*}{0.608} \\
\hline & Absent & $63(53.8 \%)$ & $54(46.2 \%)$ & $117(100.0 \%)$ & \\
\hline \multirow{2}{*}{ Hypertension } & Present & $36(50.7 \%)$ & $35(49.3 \%)$ & $71(100.0 \%)$ & \multirow{2}{*}{0.323} \\
\hline & Absent & $64(58.2 \%)$ & $46(41.8 \%)$ & $110(100.0 \%)$ & \\
\hline \multirow{2}{*}{ Ischemic heart disease } & Present & $2(25.0 \%)$ & $6(75.0 \%)$ & $8(100.0 \%)$ & \multirow{2}{*}{$0.142^{*}$} \\
\hline & Absent & $98(56.6 \%)$ & $75(43.4 \%)$ & $173(100.0 \%)$ & \\
\hline \multirow{2}{*}{ Bronchial asthma } & Present & $34(61.8 \%)$ & $21(38.2 \%)$ & $55(100.0 \%)$ & \multirow{2}{*}{0.240} \\
\hline & Absent & $66(52.4 \%)$ & $60(47.6 \%)$ & $126(100.0 \%)$ & \\
\hline
\end{tabular}

Presence of fluid on ultra sound examinations denotes the fluid leakage or the onset of critical phase of DHF. Out of 1005 patients 419 (41.7\%) showed evidence of fluid leakage suggesting DHF (Table 1). Of these patients, 131 (13\%of the total) showed evidence of leaking on the day of admission. Among DHF patients' fluid leakage was noted on the day five of fever in most (Mean $\pm S D=4.9 \pm 1.4$ days; median=5 days). NS1 antigen positivity has no association with the incidence of leaking. A separate analysis for individual co-morbidities revealed that there was no significant association between presence of hypertension, diabetes, ischemic heart disease or bronchial asthma and incidence of leaking (Table 2).

In this study onset of fluid leakage among DHF patients was noted at a mean platelet count of $33.47 \times 103$ per $\mathrm{ml}(\mathrm{SD}=18.8 \times 103$ perml). The percentage drop in the platelet count at the onset of critical phase was $30.5 \%$ ( $S D=17.7 \%)$. At the time of the onset of critical phase, themean percentage rise of PCV was $6.12 \%$ (SD=5.8\%) in DHF patients and PDW closely followed the PCV trends. It demonstrated a mean rise of $3.7 \%(S D=3.2)$ at the time of leaking (Table 4).

In contrast, in DF patients without evidence of fluid leakage, the lowest mean platelet count was $54.17 \mathrm{x}$ 103 per $\mathrm{ml}(\mathrm{SD}=24.9 \times 103$ per $\mathrm{ml})$. The analysis of serial Full Blood Counts (FBC) of DF patients revealed that the highest mean percentage drop was $15.2 \%(S D=11.8 \%)$. The highest mean percentage rise in PCV in DF patients on serial FBC done during the hospital stay was $7.9 \%$ 
Table 3.Comparison of percentage change in haematological parameters between DF and DHF patients

\begin{tabular}{|l|l|l|r|r|l|}
\hline $\begin{array}{c}\text { Change in haematological } \\
\text { parameter }\end{array}$ & Category & N & Mean (SD) & $\begin{array}{r}\text { Median } \\
\text { \{Inter-quartile (IQ) } \\
\text { Range }\end{array}$ & \multirow{2}{*}{ P value } \\
\hline \multirow{2}{*}{$\%$ Drop in platelet count } & DF & 543 & $15.2(11.8)$ & $12.3(7.0-20.6)$ & \multirow{2}{*}{0.000} \\
\cline { 2 - 5 } \% RHF & 272 & $30.5(17.8)$ & $28.2(17.7-40.8)$ & \\
\hline \multirow{2}{*}{$\%$ Rise in WCV } & DF & 358 & $7.9(4.5)$ & $7.4(4.8-10.6)$ & \multirow{2}{*}{0.000} \\
\cline { 2 - 5 } & DHF & 207 & $6.1(5.8)$ & $5.3(2.3-8.4)$ & \\
\hline & DF & 460 & $36.0(34.9)$ & $29.2(15.9-46.1)$ & \multirow{2}{*}{0.000} \\
\cline { 2 - 5 } & DHF & 214 & $26.1(22.2)$ & $21.9(10.2-33.8)$ & \\
\hline
\end{tabular}

Table 4.Comparison of haematological parameters between DHF patients who had fluid leakage on admission and who developed critical phase while in ward

\begin{tabular}{|l|l|r|r|r|c|}
\hline $\begin{array}{c}\text { Haematological } \\
\text { parameter }\end{array}$ & $\begin{array}{c}\text { Onset of } \\
\text { leaking }\end{array}$ & $\mathbf{N}$ & $\begin{array}{c}\text { Mean } \\
\text { (SD) }\end{array}$ & $\begin{array}{c}\text { Median } \\
\text { (IQ Range) }\end{array}$ & $\begin{array}{c}\text { P } \\
\text { value }\end{array}$ \\
\hline \multirow{2}{*}{ Platelet on leaking } & In Ward & 288 & $34.1(18.3)$ & $30.0(21.0-46.0)$ & \multirow{2}{*}{0.139} \\
\cline { 2 - 5 } & On Admission & 130 & $32.1(20.1)$ & $29.5(16.0-43.3)$ & \\
\hline \multirow{2}{*}{ PCV on leaking } & In Ward & 288 & $41.2(5.1)$ & $41.1(37.6-45.1)$ & \multirow{2}{*}{0.000} \\
\cline { 2 - 5 } & On Admission & 130 & $44.7(5.7)$ & $45.3(40.5-48.9)$ & \\
\hline \multirow{2}{*}{ WCC on leaking } & In Ward & 288 & $3.9(2.0)$ & $3.5(2.7-4.6)$ & \multirow{2}{*}{0.000} \\
\cline { 2 - 5 } & On Admission & 130 & $4.8(2.2)$ & $4.4(3.2-6.0)$ & \\
\hline \multirow{2}{*}{ PDW on leaking } & In Ward & 288 & $17.3(0.9)$ & $17.3(16.7-17.9)$ & \multirow{2}{*}{0.000} \\
\cline { 2 - 5 } & On Admission & 130 & $17.1(1.9)$ & $17.5(16.2-18.2)$ & \\
\hline
\end{tabular}

$(S D=4.5 \%)$. A mean rise of $26 \%(S D=22.2 \%)$ was noted in WCC in DHF patients at the time of leaking. The mean rise of WCC in DF patients on their serial FBC was $36 \%$ (SD=34.9\%). Table 3 shows the comparison of percentage changes in haematological parameters between DF and DHF patients.

As all above variables had a skewed distribution, Mann-Whitney $U$ test was performed to compare percentage changes in haematological parameters in the two categories of dengue patients which revealed that the observed differences were statistically significant.

The DHF patients in whom the fluid leakage was noted at the time of the admission were found to have a higher mean PCV (mean 44.7). This group also had a higher mean WCC and a lower mean platelet counts than the DHF patients who started their critical phase whilst being in ward.

All three parameters except platelet count on leaking demonstrated a statistically significant difference in patients who had fluid leakage on admission, compared to those who developed leakage while in ward (Table 4).

\section{Discussion}

In this study key findings include that a rapid drop in platelet counts is a reliable indication of the onset of leaking phase in DHF patients and in patients who were hospitalized during early part of the febrile period rise in PCV does not correlate with the onset of leaking. NS1 antigen positivity was not associated with disease severity and monitoring PDW did not add any useful information for clinical decision making.

In our study sample we found $41.7 \%$ patients to have DHF. This is a much higher percentage DHF than the previously reported series. In a study done in Peradeniya in central province in 2012 incidence of DHF was only 30\%.5 2017 epidemic of dengue was thought to be due to dengue type 2 (DENV2) viral infection. 6 Dengue type 2 infections are known to be associated with higher incidence of DHF. 7 The high incidence of DHF in this study was probably due to the presence of higher percentage of secondary dengue infections and the presence of DENV2. Unfortunately, we were not able to perform the viral studies and the antibody status of our patients. Majority of patients were admitted on day 4 of the illness and in DHF patients' on- 
set of critical phase was noted approximately on day 5 of the illness.

In this study NS1 antigen test was done only in $15 \%$ of patients and approximately $90 \%$ of them had a positive result. Antigen positivity had no association with the incidence of leaking. Prevalence of comorbidities hypertension, diabetes and ischemic heart disease was less as this patient population was relatively young (mean age was 34 years). We could not demonstrate a positive association with presence of comorbidities and the incidence of leaking.

Clinical management of dengue fever is currently governed by consensus guidelines based on personal experience of centers that manage large number of cases. Strong research evidence is lacking on trends in clinical parameters and amount and type of fluid requirement during critical phase. ${ }^{5}$ Clinical experience and calculated assumptions play an important role when managing patients with dengue fever in the critical phase especially in resource poor settings.

Thus, importance of detecting the onset of the critical phase is cannot be over emphasized. It can easily be done with a use of a bed side ultrasonic examination of abdomen, the facility which is not yet readily available in most hospitals in Sri Lanka. Traditionally rapid drop in platelet counts, rapid rise in PCV and rise of WCC (after serial drop) may denote the onset of fluid leakage. Presences of these previously described trends in hematological parameters were noted at the onset of the critical phase in this patient sample as well; the rise in PCV, drop in platelet counts and rise in WCC. ${ }^{3}$ Contrary to most guidelines, we did not see a $20 \%$ rise in PCV at the onset of fluid leaking. In fact, PCV rise was more in the non-leaking DF group. We have noted only a mean rise of $6.1 \%$ in PCV. This is most probably due to intravenous fluids given to the patients on admission regardless of their blood counts.

In this sample of patients, we noted a $30.5 \%$ drop in platelet counts at the onset of leaking in DHF group. Whereas in DF group, the biggest drop of platelet counts on serial counts was only $15 \%$. A precipitous drop in platelet count may denote the onset of leaking. The rapid drop in platelets more reliably predicted the onset of the critical phase than the rise in the PCV among the DHF patients who were admitted to the hospital.

However, the patients who were found to have fluid leakage on admission tend to have a higher PCV. In our study out of 419 DHF patients 131 (31.2\%) had leaking at the time of the admission to the ward. This was $13 \%$ of all the patients. Their mean PCV on admission was 44.7 and it was significantly higher than that of patients who started the critical phase after being in the ward (41.2). This may represent a higher percentage rise of PCV from the baseline and probably be closer to $20 \%$ as per traditional teaching. We did not have data of their previous PCV values to confirm that.

\section{Conclusions and recommendations}

In most DHF patients, onset of fluid leakage is seen on day 5 of the illness. In a resource poor setting if we plan an ultrasonic examination to detect fluid leaking, we should time it around day 4-5 for highest sensitivity. Contrary to the traditional teaching, 20\% PCV rise at the onset of critical phase was not seen in DHF patients in this study. Relying on a $20 \%$ rise in PCV to pick the onset of critical phase seems not reliable according to our data. In the settings where patients are being given fluids to keep them properly hydrated, we may not see a $20 \%$ rise in PCV at the time of fluid leakage. Even a small rise of $5-10 \%$ of PCV should alert the treating team of possible onset of critical phase. Precipitous drop in platelet count appears to be more reliably coincides with the onset of the critical phase. Trends in PDW closely followed the PCV and did not offer add any additional help.

Several factors need to be considered when interpreting these findings. Firstly, as we have not done serology confirmation of the dengue infection, some of the suspected febrile patients in this sample could have been infected with non dengue viral agents. Secondly, as this was a retrospective study based on a sample of patients admitted to a government hospital, the findings might not be generalizable to clinical parameters of dengue patients who are not hospitalized. Thirdly, as the analysis was based on retrieved data from hospital records, there could have been errors due to omissions and commissions in documentation.

Despite these shortcomings, we believe the study findings provide important clinical insights when managing dengue patients in resource constrained settings.

\section{Authors declare no conflicts of interests}

\section{References}

1. World Health Organization Regional office for South East Asia. Comprehensive guideline for prevention and control of dengue and dengue hemorrhagic fever revised and expanded addition. New Delhi. World Health Organization South East Asia Regional Office ;2011

2. EpidemiologyUnit,MinistryofHealth,SriLankahttp:/ /www.epid.gov.lk/web/index.php?option=com_ca sesanddeaths\&section=trends\&Itemid=448\&lang =en(accessed on 08/10/2018 
3. Ministry of Health,Sri Lanka2012. Guidelines on management of dengue fever and dengue heamorrhagic fever in adults(In collaboration with Ceylon College of Physicians).

4. Simmons CP, McPherson K, Chau NVV, Tam DTH, Young $P$, Mackenzie J, Wills $B$. Recent advances in dengue pathogenesis and clinical management. Vaccine:33(2015);7061-7068

5. Kularatne SAM, Weerakoon KGAD, Munasinghe $\mathrm{R}$, Ralapanawa UK, Pathirage M. Trends of fluid requirement in dengue fever and dengue haemorrhagic fever: a single centre experience in Sri Lanka. BMC Research Notes 2015; 8:130

6. Center for clinical management of dengue fever and dengue heamorrhagic fever. Negombo Sri Lanka(Unpublished data 2017)

7. Soo KM, Khalid B, Ching SM, Chee HY. MetaAnalysis of Dengue Severity during Infection by Different Dengue Virus Serotypes in Primary and Secondary Infections. PLoS One 2016;11(5):e 0154760 